\title{
Responses of surrogate markers of cholesterol absorption and synthesis to changes in cholesterol metabolism during various amounts of fat and cholesterol feeding among healthy men
}

\author{
Markku J. Nissinen ${ }^{1 *}$, Helena Gylling ${ }^{2}$ and Tatu A. Miettinen ${ }^{3}$ \\ ${ }^{1}$ Department of Medicine, Division of Gastroenterology, University of Helsinki, Helsinki, Finland \\ ${ }^{2}$ Department of Clinical Nutrition, University of Kuopio and Kuopio University Hospital, Kuopio, Finland \\ ${ }^{3}$ Department of Medicine, Division of Internal Medicine, University of Helsinki, Helsinki, Finland
}

(Received 18 April 2007 - Revised 6 June 2007 - Accepted 26 June 2007)

Serum ratios to cholesterol of lathosterol, and of cholestanol, campesterol and sitosterol measure respective relative cholesterol synthesis and absorption, but their clinical applicability is not known in evaluation of cholesterol metabolism under different dietary conditions. We compared relative synthesis and absorption of cholesterol to the respective absolute ones in healthy male volunteers $(n 29)$ on four subsequent diets: baseline home (HD), low-cholesterol low-fat (LCLF), high-cholesterol low-fat (HCLF) and low-cholesterol high-fat (LCHF). Serum lipids, lipoproteins, sterols, fractional cholesterol absorption and sterol synthesis were examined. HCLF and LCHF decreased fractional cholesterol absorption by approximately $23-27 \%$ from baseline HD $(P<0 \cdot 05)$ and increased the levels of total and LDL-cholesterol in serum from LCLF by approximately 9-14\% $(P<0.05)$. On HCLF, bile acid synthesis was high $(P<0.05$ for each $)$, and absolute cholesterol synthesis tended to be higher than on HD and LCHF (NS). Relative synthesis was positively associated with absolute cholesterol synthesis, but inversely with relative absorption during each diet $(P<0.05)$. The relative absorption markers were interrelated in each diet, and were also associated with fractional absorption of cholesterol in each diet but HD. In conclusion, relative markers of cholesterol absorption and synthesis reflect changes in cholesterol metabolism despite the amount of dietary fat and cholesterol consumed, but their validity with this respect is strengthened by controlled diets in metabolic studies. Additions of cholesterol and fat to a diet low in fat and cholesterol cause practically equal changes in the serum lipid profiles, whereas synthesis of cholesterol (NS) and bile acids $(P<0.05)$ were higher with the high-cholesterol feeding.

Cholesterol: Dietary cholesterol: Dietary fat: Metabolism of cholesterol: Phytosterols

Effects of dietary fats on cholesterol metabolism depend mainly on their fatty acid composition and amount of cholesterol, plant sterols, oxidized sterols and squalene ${ }^{1}$. Daily Western diets contain an average amount of $150-400 \mathrm{mg}$ (or even higher in vegetarians) of more than 200 different types of phytosterols (plant sterols and stanols), of which campesterol and sitosterol are the most abundant ones, whereas stigmasterol, avenasterol and $5 \alpha$-saturated derivatives campestanol and sitostanol form minor proportions ${ }^{2,3}$. Phytosterols are not synthesized in the human body, but they are normally absorbed to a much lesser extent than cholesterol, and, consequently, their serum levels are only $0 \cdot 1-0 \cdot 005 \%$ of total cholesterol (TC) concentration $^{4,5}$. Serum ratios of plant sterols to cholesterol depend on their dietary amount, absorption efficiency and biliary secretion. In addition, serum lipoprotein metabolism affects their absolute concentrations in serum ${ }^{6}$. Overall, the homeostatic regulation of cholesterol metabolism indicates that low intestinal absorption of cholesterol up-regulates cholesterol synthesis, whereas an increase in the intestinal cholesterol flux to the liver suppresses cholesterol synthesis ${ }^{7}$. That ratios of campesterol, sitosterol and cholestanol are related to the efficiency of intestinal cholesterol absorption among subjects with normal lipoprotein metabolism has been shown in a randomly selected male population ${ }^{8}$, and confirmed by several other studies ${ }^{6,9}$. However, their reliability with this respect has been questioned during consumption of dietary phytosterols ${ }^{10}$. Although cholestanol is a $5 \alpha$-saturated, enzymatically formed derivative of endogenous cholesterol having a low fractional absorption of about $9 \%$, it is a reliable indicator of cholesterol absorption efficiency ${ }^{9}$. In general, the serum proportions of cholesterol precursor sterols, i.e. $\Delta 8$ cholestenol, desmosterol and lathosterol, are positively related to cholesterol synthesis and negatively to those of cholestanol and plant sterols and cholesterol absorption efficiency ${ }^{8,11,12}$. Serum level of lathosterol correlates with the hepatic 3-hydroxy-3-methylglutaryl-CoA reductase activity ${ }^{13,14}$. An elevated ratio of serum lathosterol to campesterol in subjects with ileal dysfunction is suggestive of bile acid and fat

Abbreviations: HCLF, high-cholesterol low-fat diet; HD, baseline home diet; LCHF, low-cholesterol high-fat diet; LCLF, low-cholesterol low-fat diet; LDL-C, LDL-cholesterol; P/S ratio, PUFA/SFA ratio in dietary fat; TC, total cholesterol.

* Corresponding author: Dr Markku Nissinen, Biomedicum Helsinki, C422, University of Helsinki, POB 700, FI-00029 Helsinki, Finland, fax + 35894717 1851, email markku.nissinen@hus.fi and markku.nissinen@dlc.fi 
malabsorption $^{15}$. The association between increased cholesterol synthesis and serum proportions of precursor sterols is lacking among vegetarians ${ }^{16}$ and is quite modest in subjects with neomycin-induced cholesterol malabsorption ${ }^{17}$.

Recently, one focus of phytosterol research has dealt with the efficacy of dietary plant sterols and stanols in serum cholesterol lowering when given in low-fat food matrices or low-fat vehicles. However, impact of dietary cholesterol and fat, with no enrichment of dietary phytosterols, on the levels of non-cholesterol sterols in serum, and, particularly, their reciprocal associations with variables of cholesterol metabolism, are poorly known.

Aims of the present study were first to unravel how surrogate markers of cholesterol synthesis and absorption reflect changes in absolute cholesterol metabolism during diets of varying cholesterol and fat contents, and, second, to evaluate the effects of these diets on metabolism of phytosterols in healthy middle-aged male volunteers.

\section{Subjects and methods}

\section{Study population}

The study population consisted of twenty-nine healthy male subjects, mean age 54 (SEM 1) years (Table 1). They were volunteers from a randomly selected male population of about 350 individuals. The subjects studied here were given a thorough medical examination including laboratory tests and electrocardiogram at rest. No signs of diabetes or endocrinologic, liver, malignant, renal or thyroid diseases (or any other major medical problems) were detected. None had lipid-lowering medication. All subjects gave an informed consent, and the study protocol had been accepted by the Ethics Committee of the Department of Internal Medicine, Helsinki University Central Hospital.

\section{Experimental design}

Results concerning LDL kinetics, cholesterol metabolism, and serum lipids and lipoproteins have been published partially in earlier research articles ${ }^{18,19}$ and in an abstract ${ }^{20}$. The subjects were studied first at baseline on their normal dietary habits (baseline home diet, HD) and during the following three separate 6-week-long dietary periods at the outpatient ward at least 3 months apart (Table 1). On HD, their average daily cholesterol intake was $574 \mathrm{mg}$ and $38 \%$ of energy was as fat. During the second dietary period, dietary fat $(24 \%$ of energy) and cholesterol intakes $(200 \mathrm{mg} / \mathrm{d})$ were low (lowcholesterol low-fat diet, LCLF). During the third dietary period (high-cholesterol low-fat diet, HCLF), cholesterol $(890 \mathrm{mg} / \mathrm{d})$ was added to the diet as three egg yolks $/ \mathrm{d}$, and the subjects were advised to consume $30 \%$ of energy as fat. During the fourth dietary period (low-cholesterol high-fat diet, LCHF), $39 \%$ of energy was as fat, but cholesterol intake was $200 \mathrm{mg} / \mathrm{d}$. Eighteen of the study subjects attended the fourth dietary period. In this subgroup of eighteen subjects, the relative distribution of different apo E phenotypes (i.e. apo E 2, 3 and 4) was similar to that of the whole study group. The participants received written dietary instructions and oral advice from our dietitian on a weekly basis. During 1 week of each study period (the last week), the men carefully recorded their dietary intakes according to instructions given by the dietitian. Body weight was measured during each study period. Blood samples were drawn after an overnight fast once at the beginning (not shown) and three times (of which mean values were calculated) during the last week of each dietary period for lipid, lipoprotein and sterol analyses. Cholesterol absorption, sterol balance and faecal data were studied at the end of each period.

\section{Determinations}

All the analyses of serum and faecal samples were carried out from fresh samples immediately after the study.

Serum TC and TAG levels and that of HDL-cholesterol after precipitation of apo B containing-lipoproteins were determined by commercial kits (Boehringer Diagnostica, Mannheim, Germany; Wako Chemicals, Neuss, Germany). LDL-cholesterol (LDL-C) was calculated according to Friedewald et $a l .{ }^{21}$. Concentrations of cholesterol, cholestanol, cholesterol precur-

Table 1. Baseline characteristics of study subjects

(Mean values with their standard errors)

\begin{tabular}{|c|c|c|c|c|c|c|c|c|c|}
\hline \multirow[b]{3}{*}{ Variables } & \multicolumn{8}{|c|}{ Diets } & \multirow[b]{3}{*}{$P$} \\
\hline & \multicolumn{2}{|c|}{$\mathrm{HD}(n 29)$} & \multicolumn{2}{|c|}{ LCLF (n 29) } & \multicolumn{2}{|c|}{ HCLF (n 29) } & \multicolumn{2}{|c|}{ LCHF (n 18) } & \\
\hline & Mean & SEM & Mean & SEM & Mean & SEM & Mean & SEM & \\
\hline Body weight (kg) & 82 & 2 & $80^{*}$ & 2 & $80^{*}$ & 2 & $81 \ddagger$ & 3 & 0.020 \\
\hline BMI $\left(\mathrm{kg} / \mathrm{m}^{2}\right)$ & 27 & 1 & $26^{*}$ & 1 & $26^{\star}$ & 1 & $27 \ddagger$ & 1 & 0.020 \\
\hline Dietary cholesterol (mg/kg per d) & $7 \cdot 2$ & 0.4 & $2 \cdot 6^{*}$ & 0.2 & $11 \cdot 4^{\star} \dagger$ & 0.6 & $4 \cdot 1^{\star} \dagger \ddagger$ & 0.3 & $<0.001$ \\
\hline Dietary fat $(\mathrm{g} / \mathrm{kg}$ per $\mathrm{d})$ & 1.40 & 0.07 & $0.70^{*}$ & 0.05 & $0.89^{*} \dagger$ & 0.05 & $1 \cdot 25 † \ddagger$ & 0.09 & $<0.001$ \\
\hline Dietary fat (\% of energy) & 38 & 1 & $24^{\star}$ & 1 & $30 * \dagger$ & 1 & 39†‡ & 1 & $<0.001$ \\
\hline $\mathrm{P} / \mathrm{S}$ ratio & $0 \cdot 28$ & 0.02 & $0.73^{*}$ & 0.06 & $0.63^{*}$ & 0.05 & $0.51^{*} \dagger \ddagger$ & 0.04 & $<0.001$ \\
\hline Dietary campesterol (mg/kg per d)§ & 0.7 & 0.1 & 0.7 & 0.0 & 0.7 & $0 \cdot 1$ & 0.8 & 0.1 & 0.502 \\
\hline Dietary sitosterol $(\mathrm{mg} / \mathrm{kg}$ per $\mathrm{d}) \S$ & $2 \cdot 0$ & 0.1 & $2 \cdot 3$ & 0.1 & $2 \cdot 1$ & 0.2 & $2 \cdot 2$ & 0.2 & 0.683 \\
\hline
\end{tabular}

HCLF, high-cholesterol low-fat diet; HD, baseline home diet; LCHF, low-cholesterol high-fat diet; LCLF, low-cholesterol low-fat diet; P/S ratio, PUFA/SFA ratio in dietary fat.

Mean values were significantly different from those of the HD group: ${ }^{*} P<0.05$.

Mean values were significantly different from those of the LCLF group: $† P<0.05$.

Mean values were significantly different from those of the HCLF group: $\ddagger P<0.05$.

$\S n 27$ in HD. 
sor sterols $\left(\Delta^{8}\right.$-cholestenol, desmosterol and lathosterol) and plant sterols (campesterol and sitosterol) in serum were measured from non-saponifiable material by GLC, on a $50 \mathrm{~m}$ long SE-30 capillary column (Ultra 1 column, Hewlett-Packard, Palo Alto, CA, USA) with an automatic electronic integrator (Sigma 10; Hewlett-Packard) for measurement of peak areas, as described earlier ${ }^{11}$. In the following, of the cholesterol precursors only lathosterol will be dealt with. Faecal fat was measured as previously described ${ }^{22}$. Faecal bile acids and sterols (cholesterol, coprostanol, coprostanone, campesterol, sitosterol, lathosterol and cholestanol) were analysed by GLC. Faecal values of campesterol and sitosterol given in the present study include also their derivative sterols, and their respective sum represents dietary plant sterol intake.

Faecal steroid and cholesterol absorption measurements were performed as described earlier ${ }^{18,19}$. For these purposes all subjects consumed during the dietary recording week (the last week of each dietary period) a capsule containing $\left[{ }^{14} \mathrm{C}\right]$ cholesterol, $\left[{ }^{3} \mathrm{H}\right]$ sitosterol and chromic oxide $\left(\mathrm{Cr}_{2} \mathrm{O}_{3}\right)$ with each major meal (breakfast, lunch and dinner) for $7 \mathrm{~d}$. A $3 \mathrm{~d}$ stool collection was performed during the last $3 \mathrm{~d}$ of the week. Because the recoveries of $\left[{ }^{3} \mathrm{H}\right]$ sitosterol and $\mathrm{Cr}_{2} \mathrm{O}_{3}$ were identical, the faecal flow value was corrected with the recovery value of $\mathrm{Cr}_{2} \mathrm{O}_{3}{ }^{23}$. Fractional absorption for cholesterol was measured as the change in the ratio of the two isotopes ${ }^{24}$. Cholesterol synthesis was calculated by using the sterol balance technique as the difference between the sum of faecal bile acids plus neutral sterols of cholesterol origin (cholesterol + coprostanol + coprostanone) and dietary cholesterol. The latter was calculated from the dietary records (see earlier) $^{25}$. Since plant sterols are not metabolized in the human body small amounts of absorbed dietary campesterol and sitosterol are secreted through bile in intestine, indicating that their faecal amounts, including unchanged parent compounds and bacterial conversion products, represent their dietary intake. Fractional cholesterol absorption (\%) and synthesis of cholesterol $(\mathrm{mg} / \mathrm{d})$ were regarded as absolute markers of cholesterol absorption and synthesis, respectively. The corresponding relative markers for cholesterol absorption sterols were ratios of cholestanol, campesterol and sitosterol to cholesterol in serum (referred to as proportions later), whereas that of lathosterol served as a relative marker of cholesterol synthesis. In the HD, three subjects did not participate in faecal collections nor cholesterol absorption measurements.
The consumption of SFA, MUFA and PUFA was determined from the computerized data of the food records (not shown), and the ratio of PUFA to SFA (P/S ratio) was calculated for each diet.

\section{Statistical analysis}

The results are expressed as means and their standard errors. To eliminate the effect of varying cholesterol contents, serum non-cholesterol sterol values were related to serum cholesterol and are expressed in terms of $100 \times \mathrm{mmol} / \mathrm{mol}$ cholesterol of the same GLC run (referred to as proportions later). The data were analysed for significance and normality with the Number Crunching Statistical Software NCSS $^{\mathrm{TM}}$; Statistical Solutions, Kaysville, UT, USA). Logarithmic transformations were performed for skewed distributions. Comparisons between the dietary periods were performed with ANOVA for repeated measures. This was calculated for the eighteen subjects involved in each dietary period (the $\mathrm{P}$ values are given in the final column of Tables 1-4), and also separately for the whole study group, who participated in HD, LCLF and HCLF (these $P$ values are not shown). If either of the two $P$ values were below 0.05 , comparisons between the diets were carried out with Student's two-sided paired $t$ test. Analyses of covariance were studied using body weight as a covariate. Correlations were analysed by calculating Pearson's correlation test or by Spearman rank correlation test in the case of skewed distributions. To expand the range of variations the four dietary periods were combined and these data are referred to in the text as 'combined analysis' and in the tables as 'all'. $P<0.05$ was considered significant.

\section{Results}

Diets

Body weight and BMI of the study subjects were slightly lower during LCLF and HCLF than at baseline and during LCHF. Intake of campesterol and sitosterol remained equal during the dietary periods. Daily intake of dietary cholesterol was 574 (SEM 23), 208 (SEM 72), 879 (SEM 39) and 318 (SEM 20) $\mathrm{mg}$ (see Table 1 for statistical analysis) during HD, LCLF, HCLF and LCHF, respectively. As the dietary fat intake was

Table 2. Serum lipids and lipoproteins during different study periods (Mean values with their standard errors)

\begin{tabular}{|c|c|c|c|c|c|c|c|c|c|}
\hline \multirow[b]{3}{*}{ Variables } & \multicolumn{8}{|c|}{ Diets } & \multirow[b]{3}{*}{$P$} \\
\hline & \multicolumn{2}{|c|}{$\mathrm{HD}(n 29)$} & \multicolumn{2}{|c|}{ LCLF (n 29) } & \multicolumn{2}{|c|}{ HCLF (n 29) } & \multicolumn{2}{|c|}{ LCHF (n 18) } & \\
\hline & Mean & SEM & Mean & SEM & Mean & SEM & Mean & SEM & \\
\hline Total cholesterol§ (mmol/l) & $6 \cdot 01$ & $0 \cdot 21$ & $5 \cdot 07^{*}$ & 0.18 & $5 \cdot 61^{*} \dagger$ & 0.20 & $5 \cdot 55^{\star} \dagger$ & 0.27 & $<0.001$ \\
\hline HDL-cholesterol (mmol/l) & 1.41 & 0.06 & $1 \cdot 30$ & 0.05 & 1.44 & 0.06 & 1.41 & 0.08 & 0.090 \\
\hline LDL-cholesterol (mmol/l) & $3 \cdot 87$ & 0.22 & $3.04^{*}$ & 0.17 & $3 \cdot 46^{*} \dagger$ & 0.20 & $3 \cdot 32^{*} \dagger$ & 0.23 & $<0.001$ \\
\hline TAG $(\mathrm{mmol} / \mathrm{l})$ & 1.63 & 0.16 & 1.62 & 0.12 & 1.58 & 0.13 & 1.82 & 0.27 & 0.460 \\
\hline
\end{tabular}

HCLF, high-cholesterol low-fat diet; HD, baseline home diet; LCHF, low-cholesterol high-fat diet; LCLF, low-cholesterol low-fat diet. Mean values were significantly different from those of the HD group: ${ }^{*} P<0.05$.

Mean values were significantly different from those of the LCLF group: $\dagger P<0.05$. $\S$ Enzymatic cholesterol. 
Table 3. Comparison of serum non-cholesterol sterol ratios to cholesterol in study subjects during dietary periods

(Mean values with their standard errors)

\begin{tabular}{|c|c|c|c|c|c|c|c|c|c|}
\hline \multirow[b]{3}{*}{ Variables } & \multicolumn{8}{|c|}{ Diets } & \multirow[b]{3}{*}{$P$} \\
\hline & \multicolumn{2}{|c|}{$\mathrm{HD}(n 29)$} & \multicolumn{2}{|c|}{ LCLF (n 29) } & \multicolumn{2}{|c|}{ HCLF (n 29) } & \multicolumn{2}{|c|}{ LCHF (n 18) } & \\
\hline & Mean & SEM & Mean & SEM & Mean & SEM & Mean & SEM & \\
\hline Lathosterol§ & 145 & 10 & 134 & 9 & $128^{*}$ & 10 & $236^{\star} \dagger \ddagger$ & 22 & $<0.001$ \\
\hline Cholestanol§ & 85 & 6 & $119^{\star}$ & 6 & $117^{\star}$ & 5 & $133^{\star} \ddagger$ & 7 & $<0.001$ \\
\hline Campesterol§ & 170 & 14 & 183 & 15 & 169† & 15 & $247^{\star} \dagger \ddagger$ & 20 & $<0.001$ \\
\hline Sitosterol§ & 113 & 8 & $131^{*}$ & 7 & $119 \dagger$ & 8 & $137^{\star}$ & 8 & 0.041 \\
\hline
\end{tabular}

HCLF, high-cholesterol low-fat diet; HD, baseline home diet; LCHF, low-cholesterol high-fat diet; LCLF, low-cholesterol low-fat diet.

Mean values were significantly different from those of the HD group: ${ }^{*} P<0.05$.

Mean values were significantly different from those of the LCLF group: $\dagger P<0.05$

Mean values were significantly different from those of the HCLF group: $\ddagger P<0.05$

$\S 100 \times \mathrm{mmol} / \mathrm{mol}$ cholesterol.

mainly of animal origin during HD, and the amount of SFA of plant origin was high during $\mathrm{LCHF}$, the respective $\mathrm{P} / \mathrm{S}$ ratios were low.

\section{Serum and lipoprotein lipids during the diets}

The mean serum levels of TC (enzymatic) and LDL-C were lower $(P<0.05$ for each) during LCLF, HCLF and LCHF than during HD, while those of HDL-cholesterol and TAG remained stable (Table 2). The high-cholesterol and the high-fat feeding caused an increase in TC and LDL-C as compared to respective LCLF values $(P<0.05$ for both).

\section{Non-cholesterol sterols in serum and faeces}

The mean lathosterol proportions in serum decreased with the high-cholesterol feeding but profoundly increased $(P<0.05$ for other diets) with the high-fat feeding (Table 3 ), when faecal lathosterol excretion was also the highest (Table 4). Faecal excretion of cholestanol was enhanced with the highcholesterol feeding, but lower than at baseline with the highfat feeding (Table 4). The mean cholestanol, campesterol and sitosterol proportions in serum were particularly high with the high fat feeding $(P<0.05$ for each), and that of cholestanol showed elevated values also during LCLF and HCLF diets compared to HD diet $(P<0 \cdot 05$; Table 3$)$.

\section{Cholesterol absorption and metabolism}

Fractional cholesterol absorption was consistently lower during LCLF, HCLF and LCHF than HD $(P<0.05$ for each), being the lowest during HCLF $(P<0.05$ for HD and LCLF, NS for LCHF; Table 4). Cholesterol synthesis was enhanced during LCLF compared to HD $(P<0 \cdot 05$; Table 4$)$. Synthesis of bile acids was enhanced during HCLF. Excretion of faecal fat was equal during the diets, but excretion of faecal neutral sterols almost doubled with the high-cholesterol feeding as compared to LCLF and LCHF diets $(P<0.05$ for both; Table 4).

\section{Correlations between relative and absolute markers of cholesterol absorption and synthesis}

The relative cholesterol absorption markers, serum campesterol and sitosterol proportions were interrelated during each diet ( $r$ value range +0.877 to $+0.929, P<0.001$ for each). Furthermore, both plant sterol proportions correlated with serum

Table 4. Comparison of variables of cholesterol metabolism and faecal lipid excretion during different diets (Mean values with their standard errors)

\begin{tabular}{|c|c|c|c|c|c|c|c|c|c|}
\hline \multirow[b]{3}{*}{ Variables } & \multicolumn{8}{|c|}{ Diets } & \multirow[b]{3}{*}{$P$} \\
\hline & \multicolumn{2}{|c|}{$\mathrm{HD}(n 26)$} & \multicolumn{2}{|c|}{ LCLF (n 29) } & \multicolumn{2}{|c|}{ HCLF (n 29) } & \multicolumn{2}{|c|}{ LCHF (n 18) } & \\
\hline & Mean & SEM & Mean & SEM & Mean & SEM & Mean & SEM & \\
\hline Fractional cholesterol absorption (\%) & $49 \cdot 0$ & $2 \cdot 2$ & $38 \cdot 9^{*}$ & $1 \cdot 3$ & $35 \cdot 8^{*} \dagger$ & $1 \cdot 2$ & $37 \cdot 5^{\star}$ & $2 \cdot 2$ & $<0.001$ \\
\hline Cholesterol synthesis $(\mathrm{mg} / \mathrm{kg}$ per $\mathrm{d})$ & $8 \cdot 2$ & 0.8 & $11 \cdot 1^{*}$ & 0.6 & $10 \cdot 2$ & 0.9 & $9 \cdot 7$ & 0.9 & 0.067 \\
\hline Faecal bile acids (mg/kg per $\mathrm{d}$ ) & $5 \cdot 5$ & 0.3 & $5 \cdot 7$ & 0.4 & $6 \cdot 7^{*} \dagger$ & 0.5 & $5 \cdot 8 \ddagger$ & 0.7 & 0.024 \\
\hline Faecal fat $(\mathrm{g} / \mathrm{kg}$ per $\mathrm{d})$ & 0.05 & 0.00 & 0.04 & 0.00 & 0.05 & 0.00 & 0.05 & 0.00 & 0.590 \\
\hline Faecal neutral sterols (mg/kg per d) & $9 \cdot 8$ & 0.6 & $8 \cdot 0^{*}$ & 0.4 & $14 \cdot 8^{*} \dagger$ & 0.7 & $8 \cdot 0^{\star} \ddagger$ & 0.5 & $<0.001$ \\
\hline Faecal cholestanol (mg/kg per d) & $0 \cdot 16$ & 0.01 & 0.14 & 0.01 & $0.24^{*} \dagger$ & 0.01 & $0 \cdot 13^{*} \ddagger$ & 0.01 & $<0.001$ \\
\hline Faecal lathosterol (mg/kg per d) & $0 \cdot 15$ & 0.02 & 0.13 & 0.01 & $0.19^{*} \dagger$ & 0.02 & $0 \cdot 23^{*} \dagger \ddagger$ & 0.02 & $<0.001$ \\
\hline
\end{tabular}

HCLF, high-cholesterol low-fat diet; HD, baseline home diet; LCHF, low-cholesterol high-fat diet; LCLF, low-cholesterol low-fat diet.

Mean values were significantly different from those of the HD group: ${ }^{*} P<0.05$

Mean values were significantly different from those of the LCLF group: $† P<0.05$.

Mean values were significantly different from those of the HCLF group: $\ddagger P<0.05$. 
cholestanol proportions in each diet ( $r$ value range +0.492 to $+0.700, P<0.05$ for each). Serum levels of LDL-C were positively related to campesterol (HCLF and combined analysis) and sitosterol (LCLF and combined analysis) proportions in serum ( $r$ value range +0.242 to $+0.411, P<0.05$ ).

Fractional cholesterol absorption and synthesis of cholesterol were consistently inversely interrelated during each diet studied ( $r$ value range -0.377 to $-0.562, P<0.05$ for each; Table 5). Fractional absorption of cholesterol reflected consistently positively serum campesterol and sitosterol proportions with each diet, but not at baseline (Table 5; Fig. 1). However, a positive correlation with cholestanol was found solely during LCLF. Fractional absorption of cholesterol was consistently inversely associated with lathosterol in combined analysis and during the LCLF and high-cholesterol feeding (Table 5).

Synthesis of cholesterol correlated positively with the lathosterol proportion during each diet (Fig. 2). The changes in synthesis of cholesterol and lathosterol proportion between the diets correlated with each other positively only between HD and HCLF $(r+0.396, P<0 \cdot 05)$. In addition, cholesterol synthesis was almost consistently inversely associated with serum proportions of cholestanol, campesterol and sitosterol with the exception of campesterol with HCLF and cholestanol with LCHF (Table 5).

Lathosterol was consistently negatively related to serum proportions of cholestanol (Fig. 3), and with the high-cholesterol and high-fat feedings (significantly or showed a trend) to serum proportions of campesterol and sitosterol (Table 5).

The ratio of lathosterol to sitosterol was consistently positively related to the ratio of cholesterol synthesis and absorption (Fig. 4).

\section{Correlations with dietary fat, cholesterol and plant sterols}

The amount of dietary cholesterol down-regulated the synthesis of cholesterol without clear correlation to LDL-C, fractional cholesterol absorption and serum levels of sitosterol. Dietary cholesterol was positively related to serum proportions of campesterol during LCHF, with synthesis of bile acids during HCLF $(r+0 \cdot 398, P<0 \cdot 05)$ and in combined analysis $(r+0 \cdot 226, P<0 \cdot 05$; not shown $)$ and excretion of faecal neutral sterols (combined analysis), and inversely to that of lathosterol during LCHF and in combined analysis.

Dietary fat was positively correlated with campesterol and sitosterol proportions during LCLF and HCLF, with synthesis of bile acids during LCLF $(r+0.387, P<0.05)$ and HCLF $(r 0.377, P<0.05)$ and with excretion of faecal neutral sterols during LCHF (Table 6). Dietary plant sterols reflected almost consistently positively their proportions in serum during each diet. They had much lesser influence on serum proportions of lathosterol as only sitosterol intake was positively related to lathosterol at baseline, and had no detectable influence on fractional cholesterol absorption (Table 6). During the high-cholesterol feeding, dietary plant sterols were positively related to excretion of faecal neutral sterols (Table 6).

Changes in the P/S ratio from baseline to LCLF and HCLF were positively associated with respective changes of serum campesterol and sitosterol proportions ( $r$ value range +0.492 to $+0.639, P<0.02$ for each). However, neither the $\mathrm{P} / \mathrm{S}$ ratio nor amount of dietary fatty acids correlated to campesterol and sitosterol during the high-fat feeding (not shown).

\section{Discussion}

The main new results of the present study were, first, that the surrogate non-cholesterol sterol markers of cholesterol absorption and synthesis in serum preserved their sensitivity to changes in cholesterol metabolism induced by various amounts of dietary fat and cholesterol. Secondly, of the surrogate markers, lathosterol and sitosterol proportions were the most reliable ones in this respect. Thirdly, the effects of the high-fat feeding on bile acid synthesis and faecal neutral

Table 5. Correlation matrices for absolute and relative markers of cholesterol absorption and synthesis during the home diet (baseline, HD) and after switching to low-cholesterol low-fat diet (LCLF), high-cholesterol low-fat diet (HCLF) and low-cholesterol high-fat diet (LCHF), and in combined analysis

\begin{tabular}{|c|c|c|c|c|c|c|}
\hline Variables & $\begin{array}{l}\text { Synthesis of } \\
\text { cholesterol } \\
(\mathrm{mg} / \mathrm{d}) \S\end{array}$ & $\begin{array}{l}\text { Fractional cholesterol } \\
\text { absorption }(\%) \S\end{array}$ & $\begin{array}{l}\text { Lathosterol } \\
\text { (mmol/mol } \\
\text { cholesterol) }\end{array}$ & $\begin{array}{c}\text { Cholestanol } \\
\text { (mmol/mol } \\
\text { cholesterol) }\end{array}$ & $\begin{array}{c}\text { Campesterol } \\
\text { (mmol/mol } \\
\text { cholesterol) }\end{array}$ & $\begin{array}{c}\text { Sitosterol } \\
\text { ( } \mathrm{mmol} / \mathrm{mol} \\
\text { cholesterol) }\end{array}$ \\
\hline \multicolumn{7}{|c|}{ Synthesis of cholesterol $(\mathrm{mg} / \mathrm{d}) \S$} \\
\hline HD & +1.000 & $-0.487^{\star}$ & $+0.512^{\star *}$ & $-0.557^{\star *}$ & $-0.411^{*}$ & $-0.402^{*}$ \\
\hline LCLF & +1.000 & $-0.377^{\star}$ & $+0.401^{*}$ & $-0.471^{\star *}$ & $-0.504^{\star \star}$ & $-0.602^{\star \star \star}$ \\
\hline HCLF & +1.000 & $-0.413^{\star}$ & $+0.402^{*}$ & $-0.391^{\star}$ & -0.361 & $-0 \cdot 393^{\star}$ \\
\hline LCHF & +1.000 & $-0.562^{*}$ & $+0.527^{\star}$ & -0.447 & $-0 \cdot 725^{\star \star \star}$ & $-0.633^{\star \star}$ \\
\hline All & +1.000 & $-0.455^{\star \star \star}$ & $+0.313^{\star \star}$ & $-0.301^{\star *}$ & $-0.427^{\star \star \star}$ & $-0.440^{\star \star \star}$ \\
\hline \multicolumn{7}{|c|}{ Lathosterol (mmol/mol cholesterol) } \\
\hline HD & - & -0.349 & $+1 \cdot 000$ & $-0.414^{\star}$ & +0.149 & $+0 \cdot 234$ \\
\hline LCLF & - & $-0.435^{\star}$ & +1.000 & $-0.562^{\star \star \star}$ & -0.149 & -0.284 \\
\hline HCLF & - & $-0.476^{\star \star}$ & $+1 \cdot 000$ & $-0.610^{\star \star \star}$ & $-0.375^{\star}$ & $-0.383^{*}$ \\
\hline LCHF & - & -0.385 & +1.000 & $-0.744^{\star \star \star}$ & $-0.734^{\star \star \star}$ & $-0.606^{\star \star}$ \\
\hline All & - & $-0.286^{\star \star}$ & $+1 \cdot 000$ & $-0.223^{\star}$ & +0.035 & -0.080 \\
\hline \multicolumn{7}{|c|}{ Fractional cholesterol absorption (\%)§ } \\
\hline HD & - & $+1 \cdot 000$ & - & $+0 \cdot 197$ & -0.018 & +0.052 \\
\hline HCLF & - & +1.000 & - & +0.332 & $+0.422^{*}$ & $+0.489^{\star \star}$ \\
\hline LCHF & - & +1.000 & - & +0.362 & $+0.657^{\star \star}$ & $+0.635^{\star \star}$ \\
\hline All & - & +1.000 & - & +0.064 & $+0.281^{\star \star}$ & $+0.317^{\star \star}$ \\
\hline
\end{tabular}

${ }^{\star} P<0.05 ;{ }^{* \star} P<0.01 ;{ }^{* \star *} P<0.001$.

$\S n 26$ in HD. 


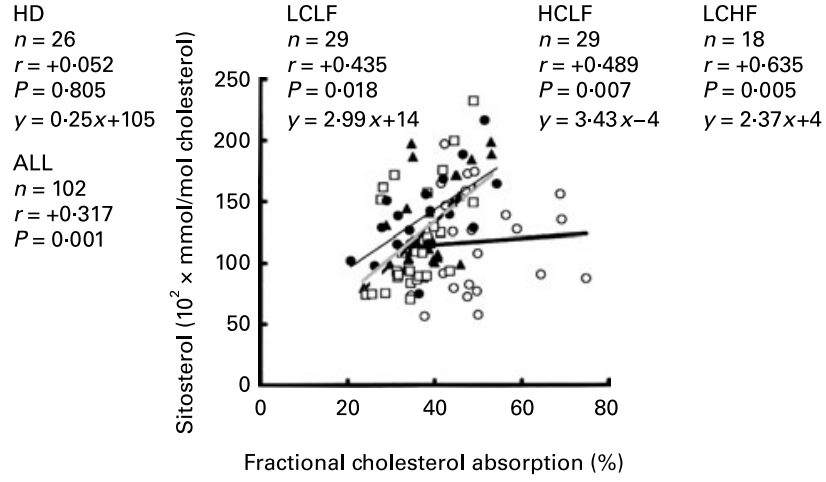

Fig. 1. Correlations between serum proportions of sitosterol and fractional cholesterol absorption during baseline home diet (HD, $\mathrm{O},-$ ), low-cholesterol low-fat diet (LCLF, $\boldsymbol{\Lambda}$, shadowed line), high-cholesterol low-fat diet (HCLF, $\square,---)$ and low-cholesterol high-fat diet (LCHF,,,- ). $r$, Spearman rank correlation coefficient.

sterol excretion differed from those of the high-cholesterol period, even though serum TC and LDL-C levels remained equal during these diets. Finally, during the high-fat diet serum lathosterol and campesterol proportions were higher than during any other dietary period. The present study employed techniques of the oral double-isotope feeding method $^{24}$ and sterol balance calculation to measure fractional cholesterol absorption and cholesterol synthesis, respectively, the golden standards of evaluating cholesterol metabolism, and usage of stable isotopes instead would have given equal results ${ }^{26,27}$.

Although the amount of dietary fat and cholesterol varied a lot between the diets administered in the present study, the intake of campesterol and sitosterol remained practically at the same level, as the maximal difference (sitosterol) between the diets was $14 \%$ (NS). This is an important aspect in evaluating changes in cholesterol metabolism as even trace amounts of dietary plant sterols may reduce cholesterol absorption and serum cholesterol levels substantially ${ }^{1}$. On the other hand, the present results revealed that the amounts of dietary campesterol and sitosterol were tightly related to their respective serum proportions in spite of their reported low fractional absorption. The present results underline the

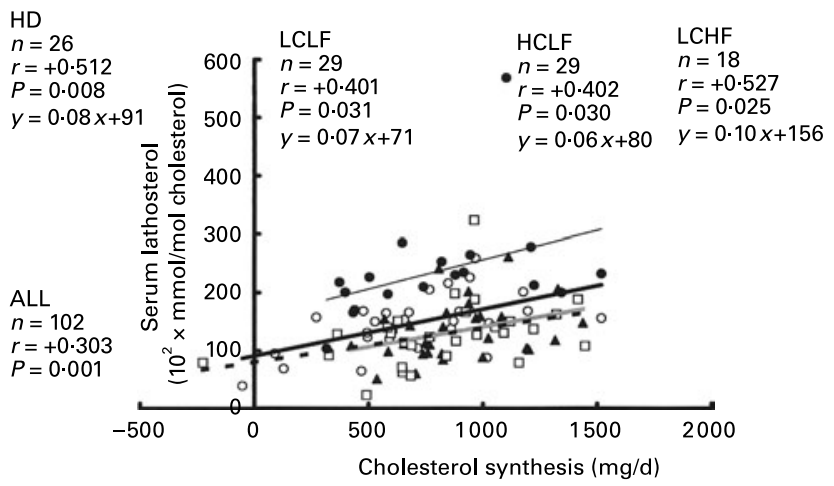

Fig. 2. Correlations between serum proportions of lathosterol and cholestero synthesis during baseline home diet $(\mathrm{HD}, \mathrm{O},-$ ), low-cholesterol low-fat diet (LCLF, $\mathbf{\Delta}$, shadowed line), high-cholesterol low-fat diet (HCLF, $\square,---)$ and low-cholesterol high-fat diet $(\mathrm{LCHF}, \mathbf{O},-)$. $r$, Spearman rank correlation coefficient.

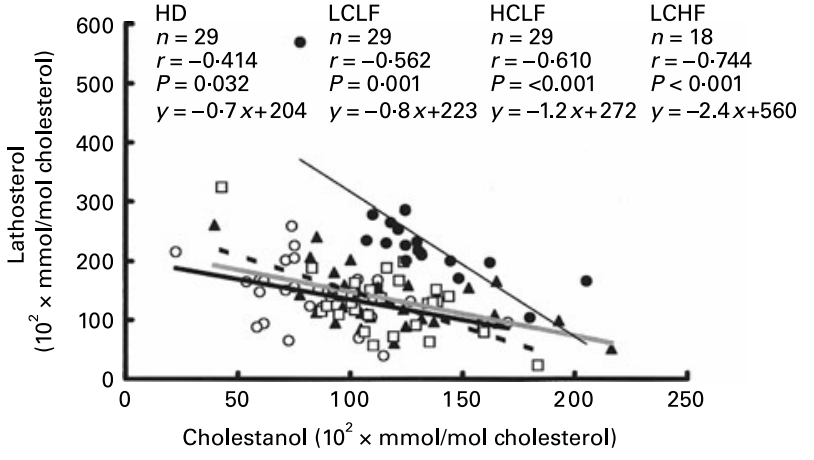

Fig. 3. Correlations between serum proportions of lathosterol and cholestanol during baseline home diet (HD, O, - ), low-cholesterol low-fat diet (LCLF, $\boldsymbol{\Delta}$, shadowed line), high-cholesterol low-fat diet (HCLF, $\square,---)$ and low-cholesterol high-fat diet (LCHF,,- ). $r$, Spearman rank correlation coefficient.

importance of controlled diets during metabolic studies in order to maintain the reliability of the surrogate markers. In addition, the results suggest that particularly the serum ratio of synthesis marker sterol to absorption marker sterol sensitively reflects changes in cholesterol metabolism. Dietary plant sterols correlated poorly with TC and LDL-C in the study subjects. The present finding that serum proportions of campesterol and sitosterol were positively related to LDL-C (both in combined analysis) is in accordance with an earlier study, which showed positive association between fractional cholesterol absorption and LDL-C ${ }^{19}$.

The present results confirmed the earlier findings that high cholesterol intake interfered with cholesterol synthesis, but, additionally we showed that the varying amount of dietary fat combined with low or high amounts of dietary cholesterol seemed also to affect the serum proportions of plant sterols. A novel finding of the present study is that, despite the high serum values of plant sterol proportions during the high-fat feeding, they were still significantly associated with changes in cholesterol metabolism. Although the variation in the serum proportions of the two plant sterols between the diets

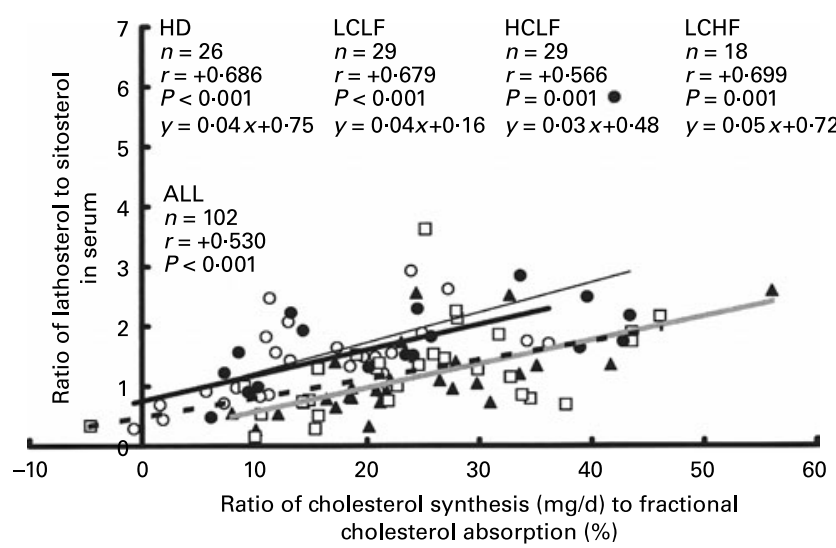

Fig. 4. Correlations between serum lathosterol to sitosterol ratios and ratios of absolute cholesterol synthesis $(\mathrm{mg} / \mathrm{d})$ to fractional cholesterol absorption in the study subjects during baseline home diet $(\mathrm{HD}, \mathrm{O},-$ ), low-cholesterol low-fat diet (LCLF, $\boldsymbol{\Delta}$, shadowed line), high-cholesterol low-fat diet (HCLF, $\square,---)$ and low-cholesterol high-fat diet (LCHF,,,-$)$. $r$, Spearman rank correlation coefficient. 
Table 6. Correlation matrices for some dietary constituents and variables of cholesterol metabolism during the home diet (baseline, HD) and after switching to low-cholesterol low-fat diet (LCLF), high-cholesterol low-fat diet (HCLF) and low-cholesterol high-fat diet (LCHF), and in combined analysis

\begin{tabular}{|c|c|c|c|c|c|c|c|}
\hline Variables & $\begin{array}{l}\text { LDL-C } \\
(\mathrm{mmol} / \mathrm{l})\end{array}$ & $\begin{array}{l}\text { Lathosterol } \\
\text { (mmol/mol } \\
\text { cholesterol) }\end{array}$ & $\begin{array}{c}\text { Campesterol } \\
\text { (mmol/mol } \\
\text { cholesterol) }\end{array}$ & $\begin{array}{c}\text { Sitosterol } \\
\text { ( } \mathrm{mmol} / \mathrm{mol} \\
\text { cholesterol) }\end{array}$ & $\begin{array}{l}\text { Fractional cholesterol } \\
\text { absorption }(\%) \S\end{array}$ & $\begin{array}{l}\text { Faecal neutral sterols } \\
\quad(\mathrm{mg} / \mathrm{kg} \text { per } \mathrm{d})\end{array}$ & $\begin{array}{c}\text { Synthesis of } \\
\text { cholesterol }(\mathrm{mg} / \mathrm{d}) \S\end{array}$ \\
\hline \multicolumn{8}{|c|}{ Dietary fat (mg/kg per d) } \\
\hline HD & +0.040 & -0.186 & +0.065 & +0.128 & +0.343 & $+0 \cdot 174$ & -0.389 \\
\hline LCLF & +0.081 & -0.041 & $+0.491^{\star *}$ & $+0.399^{*}$ & -0.002 & $+0 \cdot 130$ & -0.139 \\
\hline HCLF & +0.011 & -0.272 & $+0.455^{\star}$ & $+0.416^{\star}$ & +0.361 & $+0.452^{*}$ & -0.105 \\
\hline LCHF & -0.187 & -0.217 & +0.382 & +0.190 & -0.005 & +0.426 & -0.060 \\
\hline All & +0.173 & +0.041 & $+0.287^{\star \star}$ & +0.188 & +0.092 & +0.102 & $-0.315^{\star \star}$ \\
\hline \multicolumn{8}{|c|}{ Dietary cholesterol (mg/kg per d) } \\
\hline HD & -0.073 & -0.366 & +0.197 & $+0 \cdot 113$ & +0.112 & +0.262 & $-0.384^{*}$ \\
\hline LCLF & +0.099 & -0.148 & +0.364 & +0.339 & +0.099 & +0.174 & -0.171 \\
\hline HCLF & -0.178 & -0.189 & +0.144 & +0.115 & +0.078 & +0.352 & $-0.374^{\star}$ \\
\hline LCHF & -0.225 & $-0.558^{*}$ & $+0.573^{\star}$ & +0.393 & +0.059 & -0.044 & $-0.503^{*}$ \\
\hline All & +0.148 & $-0.239^{\star}$ & -0.063 & -0.086 & +0.021 & $+0.641^{\star \star \star}$ & $-0.224^{*}$ \\
\hline \multicolumn{8}{|c|}{ Dietary campesterol (mg/kg per d)\| } \\
\hline HD & -0.025 & +0.332 & +0.375 & +0.316 & -0.154 & -0.010 & -0.087 \\
\hline HCLF & -0.020 & -0.356 & $+0.661^{\star \star \star}$ & $+0.658^{* \star *}$ & +0.255 & $+0.523^{\star \star \star}$ & -0.147 \\
\hline LCHF & -0.085 & -0.319 & $+0.582^{*}$ & $+0.580^{\star}$ & +0.342 & +0.076 & -0.374 \\
\hline All & -0.048 & +0.013 & $+0.555^{\star \star \star}$ & $+0.511^{\star * *}$ & +0.139 & +0.039 & -0.157 \\
\hline \multicolumn{8}{|c|}{ Dietary sitosterol (mg/kg per d)\| } \\
\hline HD & -0.149 & $+0.655^{\star \star \star}$ & +0.331 & $+0.480^{\star}$ & -0.280 & +0.253 & +0.125 \\
\hline LCLF & +0.020 & $-0 \cdot 132$ & $+0.597^{\star * *}$ & $+0.647^{\star \star *}$ & +0.123 & +0.015 & +0.338 \\
\hline HCLF & -0.147 & -0.306 & $+0.518^{* *}$ & $+0.580^{\star * *}$ & +0.169 & $+0.606^{\star \star *}$ & +0.154 \\
\hline LCHF & -0.186 & -0.032 & +0.449 & $+0.478^{\star}$ & +0.140 & +0.292 & -0.186 \\
\hline All & -0.187 & +0.047 & $+0.468^{* * *}$ & $+0.541^{* \star *}$ & +0.051 & +0.098 & -0.063 \\
\hline
\end{tabular}

${ }^{*} P<0.05 ;{ }^{* *} P<0.01 ;{ }^{* \star *} P<0.001$.

$\S n 26$ in $\mathrm{HD}$.

$\| n 27$ in $\mathrm{HD}$

(HD, LCLF and HCLF) was related to the changing P/S ratio of the diets, this was not the explanation for their high proportions during the high-fat feeding. This could be explained by a slight increase (although NS) in intakes of campesterol and sitosterol, e.g. from HCLF by 10 and $5 \%$, respectively. Another explanation for this could be their better absorption during dietary consumption of high amounts of saturated fat of plant origin. This possibility is partially supported by results of a recent study ${ }^{28}$, in which hydrolysis of dietary phytosterols during their intestinal passage was more profound during a diet with normal than with low-fat consumption. This phenomenon may be due to the stimulatory effect of dietary fat on secretion and activity of pancreatic sterol ester hydrolase, which is needed in absorption of sterols ${ }^{29}$. Hydrolysis of phytosterols could promote their absorption, as sterols are mainly absorbed in unesterified form. The third explanation could be that biliary secretion of phytosterols may be lower during the high-fat consumption. There is no information whether high-fat diet interferes with the activity of the sterol transporters ABCG5 and ABCG8 and NiemannPick Like 1 protein. In a recent study in transgenic mice lacking LDL receptors the overexpression of ABCG5 and ABCG8 receptors prevented the increase of serum cholesterol during high-fat high-cholesterol diet, suggesting that diet did not interfere with these receptors ${ }^{30}$.

The correlation between the surrogate markers of cholesterol absorption and synthesis was the best between serum cholestanol and lathosterol proportions. This correlation was detected during each diet and in combined analysis, and it was convergent to those of the respective absolute markers.
However, serum campesterol and sitosterol proportions showed better correlations with the absolute markers than the cholestanol proportion did. The present results suggest that campesterol and sitosterol proportions alone as well as the serum ratio of lathosterol to cholestanol can be used to evaluate changes in absolute absorption and synthesis of cholesterol under the physiological conditions studied here. The present results were in agreement with earlier studies with respect to absolute cholesterol synthesis inversely correlating to serum cholestanol proportion ${ }^{9}$. This indicates that serum cholestanol proportion is suitable for revealing subgroups in large study populations with high or low absorption and synthesis of cholesterol.

Since high amounts of dietary plant sterols inhibit cholesterol absorption, a change in sterol balance to high cholesterol could inhibit plant sterol absorption. However, plant sterol ratios only tended to be low on HCLF. The amount of dietary cholesterol had rationale effects on the variables of cholesterol metabolism studied here: it down-regulated cholesterol synthesis, and, to a slightly lesser extent, also serum proportions of lathosterol, but, expectedly, had no association with fractional cholesterol absorption, and, as a novel finding, had only very weak influence on serum plant sterol proportions. The present findings suggest that cholesterol homeostasis is maintained during various amounts of cholesterol feeding, and that surrogate markers reliably follow up the changes in cholesterol metabolism. The present results concerning the serum lathosterol proportion are different from those of Duane $^{31}$, who reported that both lovastatin and high-cholesterol feeding (mean $1071 \mathrm{mg} / \mathrm{d}$ ) reduced markedly whole-body 
cholesterol synthesis, but only the former had a markedly reducing influence on serum lathosterol, whereas lathosterol tended even to increase with the high-cholesterol feeding. This phenomenon in Duane's study was considered to be due to high lathosterol content in five eggs consumed daily, and it was concluded that serum lathosterol does not reflect changes in cholesterol synthesis induced by dietary cholesterol $^{31}$. One reason for our different results during the high-cholesterol feeding may be the lower amount of daily dietary cholesterol and consumption of lower amount of eggs per day. The high serum proportion of lathosterol reported in the present study during the high-fat feeding may reflect its high dietary amount, which is supported by its high faecal excretion rate. However, that did not weaken its reliability as a surrogate marker within LCHF. When the change in cholesterol synthesis was compared to the change in serum lathosterol proportion between the diets, a significant positive correlation was found only between HD and HCLF. Lack of significant correlations between other diets is most probably due to low number of the study subjects and to changed metabolism of lathosterol during different diets.

Effects of dietary fat on cholesterol metabolism were similar to the high-cholesterol feeding in that serum levels of TC, LDL-C and fractional cholesterol absorption were lower as compared to the baseline. However, some differences occurred in this respect as synthesis of bile acids and excretion of faecal neutral sterols were the highest during the high-cholesterol feeding, and synthesis of cholesterol tended to be higher on HCLF than HD and LCHF.

In conclusion, the present study indicates that the homeostasis between cholesterol absorption and synthesis is consistently maintained, and that the surrogate markers of cholesterol absorption and synthesis, particularly serum lathosterol and sitosterol proportions, reflect changes in cholesterol metabolism during various intakes of cholesterol and fat among normo- or slightly hypercholesterolaemic middle-aged men. The present results emphasize the importance of controlled diets during metabolic studies in order to maintain the reliability of the surrogate markers.

\section{Acknowledgements}

This study was supported by grants from the Paavo Nurmi Foundation and the Helsinki University Central Hospital.

\section{References}

1. Ostlund RE Jr, Racette SB \& Stenson WF (2002) Effects of trace components of dietary fat on cholesterol metabolism: phytosterols, oxysterols, and squalene. Nutr Rev 60, 349-359.

2. Normen AL, Brants HAM, Voorrips LE, Andersson HA \& van den Brandt PA (2001) Plant sterol intakes and colorectal cancer risk in the Netherlands Cohort Study on Diet and Cancer. Am J Clin Nutr 74, 141-148.

3. Ostlund RE Jr (2002) Phytosterols in human nutrition. Annu Rev Nutr 22, 533-549.

4. Heinemann T, Axtmann G \& von Bergmann K (1993) Comparison of intestinal absorption of cholesterol with different plant sterols in man. Eur J Clin Invest 23, 827-831.

5. Ostlund RE Jr, McGill J, Zeng C-M, Covey DF, Stearns J, Stenson WF \& Spilburg CA (2002) Gastrointestinal absorption and plasma kinetics of soy $\Delta^{5}$-phytosterols and phytostanols in humans. Am J Physiol Endocrinol Metab 282, E911-E916.

6. Tilvis RS \& Miettinen TA (1986) Serum plant sterols and their relation to cholesterol absorption. Am J Clin Nutr 43, 92-97.

7. Grundy SM, Ahrens EH \& Davignon J (1969) The interaction of cholesterol absorption and cholesterol synthesis in man. J Lipid Res 10, 304-315.

8. Miettinen TA, Tilvis RS \& Kesäniemi YA (1990) Serum plant sterols and cholesterol precursors reflect cholesterol absorption and synthesis in volunteers of a randomly selected male population. Am J Epidemiol 131, 20-31.

9. Miettinen TA, Tilvis RS \& Kesäniemi YA (1989) Serum cholestanol and plant sterol levels in relation to cholesterol metabolism in middle-aged men. Metabolism 38, 136-140.

10. Vanstone CA \& Jones PJH (2004) Limitations of plasma plant sterols as indicators of cholesterol absorption. Am J Clin Nutr 79, 340-341.

11. Miettinen TA \& Koivisto P (1983) Non-cholesterol sterols and bile acid production in hypercholesterolaemic patients with ileal by-pass. In Bile Acids and Cholesterol in Health and Disease, pp. 183-187 [G Paumgartner, A Stiehl and W Gerok, editors]. Lancaster, PA: MTP Press.

12. Miettinen TA (1985) Cholesterol precursors and their diurnal rhythm in lipoproteins of patients with jejuno-ileal bypass and ileal dysfunction. Metabolism 34, 425-430.

13. Björkhem I, Miettinen TA, Reihner E, Ewerth S, Angelin B \& Einarsson K (1987) Correlation between serum levels of some cholesterol precursors and activity of HMG-CoA reductase in human liver. J Lipid Res 28, 1137-1143.

14. Kempen HJM, Glatz JFC, Leuven JAG, van der Voort HA \& Katan MB (1988) Serum lathosterol concentration is an indicator of whole-body cholesterol synthesis in humans. J Lipid Res 29, 1149-1155.

15. Färkkilä MA \& Miettinen TA (1988) Plasma lathosterol and campesterol in detection of ileal dysfunction. Scand J Gastroenterol 23, 19-25.

16. Vuoristo M \& Miettinen TA (1994) Absorption, metabolism and serum concentrations of cholesterol in vegetarians: effects of cholesterol feeding. Am J Clin Nutr 58, 1325-1331.

17. Miettinen TA (1979) Effects of neomycin alone and in combination with cholestyramine on serum cholesterol and fecal steroids in hypercholesterolemic subjects. J Clin Invest 64, $1485-1493$

18. Gylling H \& Miettinen TA (1992) Cholesterol absorption and synthesis related to low density lipoprotein metabolism during varying cholesterol intake in men with different apoE phenotypes. J Lipid Res 33, 1361-1371.

19. Miettinen TA, Gylling H, Vanhanen H \& Ollus A (1992) Cholesterol absorption, elimination, and synthesis related to LDL kinetics during varying fat intake in men with different apoprotein E phenotypes. Arterioscler Thromb 12, 1044-1052.

20. Gylling H \& Miettinen TA (1991) High cholesterol and fat intake have similar respective effects on serum cholesterol, LDL kinetics and cholesterol metabolism (abstract). Circulation 84(4), Suppl. II, 680.

21. Friedewald WT, Levy RI \& Fredrickson DS (1972) Estimation of the concentration of low density lipoprotein in plasma, without use of the preparative ultracentrifuge. Clin Chem 18, 499-502.

22. van de Kamer JH, Bokkel Huinink HT \& Weyers HA (1949) Rapid method for the determination of fat in feces. $J$ Biol Chem 177, 347-355.

23. Bolin DW, King RP \& Klosterman EW (1952) A simplified method for the determination of chromic oxide $\left(\mathrm{Cr}_{2} \mathrm{O}_{3}\right)$ when used as an index substance. Science 116, 634-635.

24. Crouse JR \& Grundy SM (1978) Evaluation of a continuous isotope feeding method for measurement of cholesterol absorption in man. J Lipid Res 19, 221-232. 
25. Ahlström A, Räsänen L \& Kuvaja K (1972) A method of data processing for food consumption surveys. Ann Acad Sci Fenn (Biol) 194, 1-8.

26. Bosner MS, Ostlund RE Jr, Osofisan O, Grosklos J, Fritschle C \& Lange LG (1993) Assessment of percent cholesterol absorption in humans with stable isotopes. J Lipid Res 34, 1047-1053.

27. Lütjohann D, Meese CO, Crouse JR III \& von Bergmann K (1993) Evaluation of deuterated cholesterol and deuterated sitostanol for measurement of cholesterol absorption in humans. J Lipid Res 34, 1039-1046.

28. Nissinen MJ, Gylling H \& Miettinen TA (2006) Effects of plant stanol esters supplied in a fat free milieu by pastilles on cholesterol metabolism in colectomized human subjects. Nutr Metab Cardiovasc Dis 16, 426-435.

29. Howles PN, Carter CP \& Hui DY (1996) Dietary free and esterified cholesterol absorption in cholesterol esterase (bile salt-stimulated lipase) gene-targeted mice. J Biol Chem 271, 7196-7202.

30. Wilund KR, Yu L, Xu F, Hobbs HH \& Cohen JC (2004) Highlevel expression of ABCG5 and ABCG8 attenuates dietinduced hypercholesterolemia and atherosclerosis in $\mathrm{Ldl}^{-1-}$ mice. J Lipid Res 45, 1429-1436.

31. Duane WC (1995) Serum lathosterol levels in human subjects reflect changes in whole body cholesterol synthesis induced by lovastatin but not dietary cholesterol. J Lipid Res 36, 343-348. 